\title{
Statement on guidance for genetic counseling in advanced paternal age
}

\author{
Helga V. Toriello, $P h D^{1}$, and Jeanne M. Meck, $P h D^{2}$, for the Professional Practice and Guidelines Committee
}

Key Words: paternal age, genetic counseling, mutation, chromosome anomalies

\begin{abstract}
Disclaimer: This guideline is designed primarily as an educational resource for healthcare providers to help them provide quality medical genetic services. Adherence to this guideline does not necessarily assure a successful medical outcome. This guideline should not be considered inclusive of all proper procedures and tests or exclusive of other procedures and tests that are reasonably directed to obtaining the same results. In determining the propriety of any specific procedure or test, the geneticist should apply his or her own professional judgment to the specific clinical circumstances presented by the individual patient or specimen. It may be prudent, however, to document in the patient's record the rationale for any significant deviation from this guideline.
\end{abstract}

In 1996, a practice guideline on genetic counseling for advanced paternal age was published. The current document updates the state of knowledge of advanced paternal age effects on single gene mutations, chromosome anomalies, and complex traits. Genet Med 2008:10(6):457-460.

There is no clearly accepted definition of advanced paternal age. A frequently used criterion is any man aged 40 years or older at the time of conception. The current population mean paternal age is 27 years.

Advanced paternal age is associated with an increased risk of new gene mutations. Because of the large number of cell divisions during spermatogenesis, the mutation rate for base substitutions is much higher in men than women, and increases with paternal age. The risk for genetic defects increases linearly for some conditions, and exponentially for others. ${ }^{1-3}$ The conditions most strongly associated with advanced paternal age are those caused by mutations in the form of single base substitutions in the FGFR2, FGFR3, and RET genes, and include Pfeiffer syndrome, Crouzon syndrome, Apert syndrome, achondroplasia, thanatophoric dysplasia, as well as MEN2A and MEN2B. ${ }^{4}$ Some dominant conditions that are caused by gene changes that include both point mutations and base pair deletions (e.g., neurofibromatosis) show a lesser association with paternal age. Other dominant conditions show no association with increased paternal age. ${ }^{5}$ Although Friedman ${ }^{6}$ had estimated that the risk for autosomal dominant disorders af-

From the ${ }^{1}$ Genetics Services, Spectrum Health, Grand Rapids, Michigan; and ${ }^{2}$ Quest Diagnostics/Nichols Institute, Inc. Chantilly, Virginia.

Helga V. Toriello, PhD, Genetics Services, Spectrum Health, 21 Michigan St, Ste 465, Grand Rapids, MI 49503-2528. E-mail: helga.toriello@spectrum-health.org.

Approved by the Board of Directors July 28, 2007.

Go to www.geneticsinmedicine.org for a printable copy of this document.

American College of Medical Genetics, 9650 Rockville Pike, Bethesda, MD 20814

Submitted for publication March 4, 2008.

Accepted for publication March 5, 2008.

DOI: 10.1097/GIM.0b013e318176fabb fecting offspring of fathers aged 40 or more was $0.3-0.5 \%$, it is now thought that the actual risk is lower. ${ }^{7}$ There is also a growing body of evidence that advanced paternal age is associated with an increased risk for complex disorders such as some congenital anomalies, schizophrenia, autism spectrum disorders, and some forms of cancer. ${ }^{8-12}$ For most conditions the relative risk is two or less. However, the mechanism for the increased risk is unknown, and in some cases, the observed paternal age effect may be an artifact of some other causative factor.

In general, for autosomes and sex chromosomes, there is no compelling evidence that chromosomal aberrations (aneuploidy or structural chromosome abnormalities) are significantly increased in newborns as paternal age increases. The low incidence of paternally derived extra chromosomes in trisomies combined with the relatively small number of children fathered by older men makes it difficult to demonstrate a paternal age effect. Two possible exceptions are trisomy 21 and Klinefelter syndrome. Recent data on Down syndrome suggest a paternal age effect, either acting alone or in combination with a maternal age effect. ${ }^{13,14}$ This observation is supported by reports of increased aneuploidy rates in sperm for some of the chromosomes, including 21 and the sex chromosomes. ${ }^{15-17}$ In summary, there is a wide range of genetic disorders that may be related to advanced paternal age (Table 1). Overall, it seems that the risk of birth defects and some chromosome disorders may be minimally increased, and the risk for later onset disorders may also show a small increase with advanced paternal age. There are currently no screening or diagnostic test panels which specifically target those conditions that increase with paternal age. If the older male's partner is currently pregnant, the pregnancy should be treated as any other according to pre- 
Table 1

Paternal age risks

\begin{tabular}{|c|c|c|c|c|c|c|}
\hline Type & Specific condition & $\begin{array}{l}\text { Age } \\
\text { (relative to } \\
\text { reference age) }\end{array}$ & $\begin{array}{c}\text { Relative risk } \\
\text { (CI, if available) }\end{array}$ & $\begin{array}{l}\text { Population risk } \\
\text { (or reference risk) }\end{array}$ & Adjusted risk & $\begin{array}{l}\text { References } \\
\text { (first author's } \\
\text { name only) }\end{array}$ \\
\hline \multirow{20}{*}{$\begin{array}{l}\text { Autosomal } \\
\text { dominant }\end{array}$} & \multirow[t]{6}{*}{ Achondroplasia } & $>50(25-29)$ & 7.8 & \multirow[t]{6}{*}{$1 / 15,000$} & $1 / 1923$ & Risch $^{1}$ \\
\hline & & $30-34(<20)$ & 3.5 & & $1 / 4285$ & \multirow[t]{5}{*}{ Tiemann-Boege ${ }^{21}$} \\
\hline & & $35-39(<20)$ & 4 & & $1 / 3750$ & \\
\hline & & $40-44(<20)$ & 8 & & $1 / 1875$ & \\
\hline & & $45-49(<20)$ & 9 & & $1 / 1666$ & \\
\hline & & $50-54(<20)$ & 12 & & $1 / 1250$ & \\
\hline & Apert & $>50(25-29)$ & 9.5 & $1 / 50,000$ & $1 / 5263$ & Risch $^{1}$ \\
\hline & Pfeiffer & $>50(25-29)$ & 6 & $1 / 100,000$ & $1 / 16,666$ & Glaser $^{22}$ \\
\hline & Crouzon & $>50(25-29)$ & 8 & $1 / 50,000$ & $1 / 6250$ & \\
\hline & Progeria & Unknown & Effect seen & "Exceedingly rare" & & \\
\hline & MEN2A & Unknown & Effect seen & $1 / 30,000$ & & \\
\hline & MEN2B & Unknown & Effect seen & $1 / 30,000$ & & \\
\hline & \multirow[t]{2}{*}{ Neurofibromatosis I } & $>50(25-29)$ & $3.7^{a}$ & \multirow[t]{2}{*}{$1 / 3000-1 / 4000$} & $1 / 810-1 / 1080$ & Risch $^{1}$ \\
\hline & & $>40(<30)$ & 2.9 & & $1 / 1034-1 / 1380$ & Bunin $^{23}$ \\
\hline & \multirow{2}{*}{$\begin{array}{l}\text { Osteogenesis } \\
\text { imperfecta }\end{array}$} & $>35(<25)$ & 2.5 & \multirow[t]{2}{*}{$1 / 10,000$} & $1 / 4000$ & Carothers ${ }^{24}$ \\
\hline & & $>35(<35)$ & $1.37(0.73-6.89)$ & & $1 / 7300$ & Orioli ${ }^{25}$ \\
\hline & $\begin{array}{l}\text { Thanatophoric } \\
\text { dysplasia }\end{array}$ & $>35(<35)$ & $3.18(1.48-6.89)$ & $1 / 20,000-1 / 50,000$ & $1 / 6290-1 / 15,723$ & Orioli $^{25}$ \\
\hline & \multirow[t]{3}{*}{ Retinoblastoma } & $>45$ & $3^{a}(0.21-41.7)$ & \multirow[t]{3}{*}{$1 / 15,000-1 / 20,000$} & $1 / 5000-1 / 6667$ & Dockerty, Yip ${ }^{26,27}$ \\
\hline & & $>35(<35)$ & $1.34(1.04-1.74)$ & & $1 / 11,200-1 / 14,925$ & $\mathrm{Moll}^{28}$ \\
\hline & & $>50(32.5)$ & 5 & & $1 / 3000-1 / 4000$ & DerKinderen $^{29}$ \\
\hline \multirow[t]{9}{*}{ Chromosomal } & \multirow[t]{8}{*}{ Down syndrome } & $40-44(20-29)$ & $1.37(0.48-3.86)$ & \multirow{3}{*}{$\begin{array}{c}1 / 1200 \\
\text { (mat. age } 20-29)\end{array}$} & $1 / 876$ & \multirow[t]{3}{*}{$\mathrm{Zhu}^{30}$} \\
\hline & & $45-49(20-29)$ & $2.68(0.76-9.51)$ & & $1 / 448$ & \\
\hline & & $>49(20-29)$ & $4.5(1.0-20.3)$ & & $1 / 267$ & \\
\hline & & $40-44(25-29)$ & $1.45(1.26-1.68)$ & \multirow{5}{*}{$\begin{array}{l}\text { Use maternal age } \\
\text { as baseline for } \\
\text { counseling } \\
\text { purposes }^{b}\end{array}$} & & \multirow[t]{3}{*}{ Yang 31} \\
\hline & & $45-49(25-29)$ & $1.28(1.04-1.57)$ & & & \\
\hline & & $>49(25-29)$ & $1.39(1.04-1.83)$ & & & \\
\hline & & None given & "May be increased" & & & Kuhnert $^{16}$ \\
\hline & & None given & $\begin{array}{l}\text { "Paternal age effect in } \\
\text { association with maternal } \\
\text { age }(>35) \text { effect" }\end{array}$ & & & Fisch $^{14}$ \\
\hline & Klinefelter syndrome & $>50$ (20’s) & $1.6^{c}(0.69-3.0)$ & $1 / 500$ men & $1 / 312$ men & Lowe $^{32}$ \\
\hline \multirow{3}{*}{$\begin{array}{l}\text { Congenital } \\
\text { anomalies }\end{array}$} & VSD & $>40(<40)$ & $1.69^{a}$ & $1 / 200$ & $1 / 118$ & Olshan ${ }^{33}$ \\
\hline & ASD & $>35$ & $1.95^{a}$ & $1 / 400$ & $1 / 205$ & $\operatorname{Lian}^{11}$ \\
\hline & $\begin{array}{l}\text { Tracheoesophageal } \\
\text { fistula }\end{array}$ & $>50(25-29)$ & $2.55(1.28-4.6)$ & $1 / 3600$ & $1 / 1412$ & Yang ${ }^{31}$ \\
\hline \multirow{5}{*}{$\begin{array}{l}\text { Other complex } \\
\text { disorders }\end{array}$} & \multirow[t]{2}{*}{ Childhood leukemia } & $>35$ & 1.5 & \multirow[t]{2}{*}{$1 / 25,000$} & $1 / 16,667$ & Murray $^{34}$ \\
\hline & & $>40(<25)$ & $1.14(0.85-1.53)$ & & $1 / 21930$ & Yip $^{27}$ \\
\hline & \multirow{3}{*}{$\begin{array}{l}\text { Childhood CNS } \\
\text { tumor }\end{array}$} & $30-34(<25)$ & $1.34(1.04-1.72)$ & \multirow[t]{3}{*}{$1 / 36,000$} & $1 / 26,866$ & \multirow[t]{3}{*}{ Yip $^{27}$} \\
\hline & & $35-39(<25)$ & $1.4(1.04-1.86)$ & & $1 / 25,714$ & \\
\hline & & $>40(<25)$ & $1.69(1.21-2.35)$ & & $1 / 21,302$ & \\
\hline
\end{tabular}


Table 1

(Continued)

\begin{tabular}{|c|c|c|c|c|c|c|}
\hline Type & Specific condition & $\begin{array}{c}\text { Age } \\
\text { (relative to } \\
\text { reference age) }\end{array}$ & $\begin{array}{c}\text { Relative risk } \\
\text { (CI, if available) }\end{array}$ & $\begin{array}{l}\text { Population risk } \\
\text { (or reference risk) }\end{array}$ & Adjusted risk & $\begin{array}{l}\text { References } \\
\text { (first author's } \\
\text { name only) }\end{array}$ \\
\hline & $\begin{array}{l}\text { Childhood type } 1 \\
\text { diabetes }\end{array}$ & $>34(<25)$ & $1.52(1.1-2.09)$ & $1 / 415$ & $1 / 273$ & Cardwell ${ }^{35}$ \\
\hline & \multirow[t]{2}{*}{ Epilepsy } & $35-39$ & $1.18(1.02-1.26)$ & \multirow[t]{2}{*}{$1 / 100$} & $1 / 85$ & \multirow[t]{2}{*}{ Vestergaard ${ }^{36}$} \\
\hline & & $40-45$ & $1.3(1.08-1.55)$ & & $1 / 770$ & \\
\hline & \multirow[t]{6}{*}{ Schizophrenia } & $>50(20-24)$ & $4.62(2.28-9.36)$ & \multirow[t]{6}{*}{$1 / 100$} & $1 / 22$ & Rasmussen $^{37}$ \\
\hline & & $35-44(15-24)$ & $1.6(1.0-2.6)$ & & $1 / 62.5$ & \multirow[t]{3}{*}{ Zammit $^{38}$} \\
\hline & & $45-54(15-24)$ & $1.6(0.8-3.1)$ & & $1 / 62.5$ & \\
\hline & & $>54(15-24)$ & $3.8(1.3-11.8)$ & & $1 / 26$ & \\
\hline & & $>49(<25)$ & 3 & & $1 / 33$ & Malaspina $^{12}$ \\
\hline & & $>32(<28)$ & $3(1.49-6.04)$ & & $1 / 33$ & Tsuchiya $^{39}$ \\
\hline & \multirow[t]{2}{*}{ Autism } & $>40(<30)$ & $5.75(2.65-12.46)$ & \multirow[t]{2}{*}{$1 / 1000$} & \multirow[t]{2}{*}{$1 / 174$} & Reichenberg ${ }^{40}$ \\
\hline & & Unknown & Effect seen & & & Cantor ${ }^{9}$ \\
\hline & \multirow{2}{*}{$\begin{array}{l}\text { Autism spectrum } \\
\text { disorders }\end{array}$} & $35-39(25-29)$ & $1.38(1.04-1.84)$ & \multirow[t]{2}{*}{$1 / 200$} & $1 / 145$ & \multirow[t]{2}{*}{ Croen $^{41}$} \\
\hline & & $>39(25-29)$ & $1.52(1.1-2.1)$ & & $1 / 131$ & \\
\hline & Breast cancer & $>40(<30)$ & $1.6(1.04-2.32)$ & $1 / 8.5$ & $1 / 5.3$ & Choi $^{42}$ \\
\hline & Prostate cancer & $>38(<27)$ & $1.7(1.0-2.8)$ & $1 / 5.9$ & $1 / 3.5$ & Zhang ${ }^{43}$ \\
\hline & Multiple sclerosis & $51-55(21-25)$ & $2.0(1.35-2.96)$ & & & Montgomery ${ }^{44}$ \\
\hline \multirow[t]{6}{*}{ Other } & \multirow{2}{*}{$\begin{array}{l}\text { Spontaneous } \\
\text { miscarriages }\end{array}$} & $>35(<35)$ & $1.26(1.0-1.6)$ & \multirow[t]{2}{*}{$1 / 7$} & $1 / 5.3$ & Slama ${ }^{45}$ \\
\hline & & $>39(25-29)$ & $1.6(1.2-2.0)$ & & $1 / 4$ & Kleinhaus ${ }^{46}$ \\
\hline & Relative infertility & $>39(<39)$ & $2.3(1.67-3.17)$ & $1 / 14$ couples & $1 / 6.2$ & De la Rochebrochard ${ }^{47}$ \\
\hline & Low birth weight & $>34(20-34)$ & $1.7(1.3-2.2)$ & $1 / 40$ & $1 / 23$ & Reichman $^{48}$ \\
\hline & \multirow[t]{2}{*}{ Preeclampsia } & $35-44(25-34)$ & $1.24(1.05-1.46)$ & $1 / 62$ & $1 / 50$ & \multirow[t]{2}{*}{ Harlap ${ }^{49}$} \\
\hline & & $>44(25-34)$ & $1.8(1.04-1.51)$ & $1 / 62$ & $1 / 34$ & \\
\hline \multirow[t]{2}{*}{ Total risk } & \multirow{2}{*}{$\begin{array}{l}\text { For } 86 \text { examined } \\
\text { congenital } \\
\text { anomalies }\end{array}$} & $>40(<20)$ & 1.2 & \multirow[t]{2}{*}{$1 / 50$} & $1 / 42$ & \multirow[t]{2}{*}{$\operatorname{Lian}^{11}$} \\
\hline & & $>50(<20)$ & 1.3 & & $1 / 38$ & \\
\hline
\end{tabular}

This table is meant to show the findings of various studies examining the effect of paternal age on the condition in question. It is not meant to be a comprehensive guide to counseling, but to merely indicate conditions which have been studied and results obtained from those studies.

${ }^{a}$ Increased risk not shown by other studies.

${ }^{b}$ Suggestion for this adjustment made by the author of this document. There are no data regarding use of paternal age for counseling for serum screening results.

${ }^{c}$ Based on frequency of XY sperm.

natal diagnosis guidelines established by the American College of Medical Genetics and American College of Obstetricians and Gynecologists, ${ }^{18-20}$ with the prenatal counseling session including a discussion about the potentially increased risk of Down syndrome attributable to increased paternal age. Because of this and the possibility of ultrasound detection of some of the features of the autosomal dominant conditions noted above (e.g., thanatophoric dysplasia), an ultrasound is recommended at 18-20-weeks gestation to evaluate fetal growth and development. However, it is unlikely to detect many of the conditions of interest. Prospective couples should receive individualized genetic counseling to address specific concerns.

\section{RESOURCES}

1. Archer NP, Langlois PH, Suarez L, Brender J, et al. Association of paternal age with prevalence of selected birth defects. Birth Defects Res A Clin Mol Teratol 2007; 79:27-34.

2. Cedergren MI, Selbing AJ, Kallen BA. Risk factors for cardiovascular malformation-a study based on prospectively collected data. Scand J Work Environ Health 2002;28:12-17.

3. Dzurova D, Pikhart H. Down syndrome, paternal age and education: comparison of California and the Czech Republic. BMC Public Health 2005;5:69. 
4. MacDonald M, Hassold T, Harvey J, Wang LH, et al. The origin of 47, XXY and 47, XXX aneuploidy: heterogeneous mechanisms and the role of aberrant recombination. Hum Mol Genet 1994;3:1365-1371.

5. Sherman SL, Freeman SB, Allen EG, Lamb NE. Risk factors for nondisjunction of Trisomy 21. Cytogenetic Genome Res 2005;111:273-280.

6. Xue F, Colditz GA, Willett WC, Rosner BA, et al. Parental age at delivery and incidence of breast cancer: a prospective cohort study. Breast Cancer Res Treat 2007;104:331340 .

\section{References}

1. Risch N, Reigh EW, Wishnick MW, McCarthy JG. Spontaneous mutation and parental age in humans. Am J Hum Genet 1987;41:218-248.

2. Crow JF. The high spontaneous mutation rate: is it a health risk? Proc Natl Acad Sci U S A 1997;94:8380-8386.

3. Crow JF. Age and sex effects on human mutation rates: an old problem with new complexities. J Radiat Res 2006;47(suppl):B72-B82.

4. Jung A, Schuppe HC, Schill WB. Are children of older fathers at risk for genetic disorders? Andrologia 2003;35:191-199.

5. Splendore A, Jabs EW, Felix TM, Passos-Bueno MR. Parental origin of mutations in sporadic cases of Treacher Collins syndrome. Eur J Hum Genet 2003;11:718-722.

6. Friedman JM. Genetic disease in the offspring of older fathers. Obstet Gynecol 1981; 57:745-749.

7. Hook EB. Paternal age and effects on chromosomal and specific locus mutations and on other genetic outcomes in offspring. In: Mastroianni L, Paulsen CA, editors. Aging, reproduction, and the climacteric. New York: Plenum Press, 1986:117-145.

8. Byrne M, Agerbo E, Ewald H, Eaton WW, et al. Parental age and risk of schizophrenia: a case-control study. Arch Gen Psychiatry 2003;60:673-678.

9. Cantor RM, Yoon JL, Furr J, Lajonchere CM. Paternal age and autism are associated in a family-based sample. Mol Psychiatr 2007;12:419-421.

10. Dalman C, Allebeck P. Paternal age and schizophrenia: further support for an association. Am J Psychiatry 2002;159:1591-1592.

11. Lian ZH, Zack MM, Erickson JD. Paternal age and the occurrence of birth defects. Am J Hum Genet 1986;39:648-660.

12. Malaspina D, Harlap S, Fennig S, Heiman D, et al. Advancing paternal age and the risk of schizophrenia. Arch Gen Psychiatry 2001;58:361-367.

13. Shi Q, Martin RH. Aneuploidy in human sperm: a review of the frequency and distribution of aneuploidy, effects of donor age and lifestyle factors. Cytogenet Cell Genet 2000;90:219-226.

14. Fisch H, Hyun G, Golden R, Hensle TW, et al. The influence of paternal age on Down syndrome. J Urol 2003;169:2275-2278.

15. Sartorelli EM, Mazzucatto LF, de Pina-Neto JM. Effect of paternal age on human sperm chromosomes. Fertil Steril 2001;76:1119-1123.

16. Kuhnert B, Nieschlag E. Reproductive functions of the ageing male. Hum Reprod Update 2004;10:327-339.

17. Buwe A, Guttenbach M, Schmid M. Effect of paternal age on the frequency of cytogenetic abnormalities in human spermatozoa. Cytogenet Genome Res 2005;11: 213-228.

18. ACOG Practice Bulletin. Clinical management guidelines for obstetrician-gynecologists. Ultrasonography in pregnancy. No. 58. Obstet Gynecol 2004;104:1449-1458.

19. ACOG Practice Bulletin. Screening for fetal chromosomal abnormalities. No. 77. Obstet Gynecol 2007;109:217-227.

20. Driscoll DA, Gross SJ, ACMG Professional Practice and Guidelines Committee. First trimester diagnosis and screening for fetal aneuploidy. Genet Med 2008; 10: $73-75$.

21. Tiemann-Boege I, Navidi W, Grewal R, Cohn D, et al. The observed human sperm mutation frequency cannot explain the achondroplasia paternal age effect. Proc Natl Acad Sci U S A 2002;99:14952-14957.
22. Glaser RL, Jabs EW. Dear old dad. Sci Aging Knowledge Environ 2004;21:2004:re1.

23. Bunin GR, Needle M, Riccardi VM. Paternal age and sporadic neurofibromatosis 1 : a case-control study and consideration of the methodologic issues. Genet Epidemio 1997;14:507-516.

24. Carothers AD, McAllion SJ, Paterson CR. Risk of dominant mutation in older fathers: evidence from osteogenesis imperfecta. J Med Genet 1986;23:227-230.

25. Orioli IM, Castilla EE, Scarano G, Mastroiacovo P. Effect of paternal age in achondroplasia, thanatophoric dysplasia, and osteogenesis imperfecta. Am J Med Genet 1995;59:209-217.

26. Dockerty JD, Draper G, Vincent T, Rowan SD, et al. Case-control study of parental age, parity and socioeconomic level in relation to childhood cancers. Int J Epidemio 2001;30:1428-1437.

27. Yip BH, Pawitan Y, Czene K. Parental age and risk of childhood cancers: a population-based cohort study from Sweden. Int J Epidemiol 2006;35:1495-1503.

28. Moll AC, Imhof SM, Kuik DJ, Bouter LM, et al. High parental age is associated with sporadic hereditary retinoblastoma: the Dutch retinoblastoma register 1862-1994. Hum Genet 1996;98:109-112.

29. DerKinderen DJ, Koten JW, Tan KE, Beemer FA, et al. Parental age in sporadic hereditary retinoblastoma. Am J Ophthalmol 1990;110:605-609.

30. Zhu JL, Madsen KM, Vestergaard M, Olesen AV, et al. Paternal age and congenital malformations. Hum Reprod 2005;20:3173-3177.

31. Yang Q, Wen SW, Leader A, Chen XK, et al. Paternal age and birth defects: how strong is the association? Hum Reprod 2007;22:696-701.

32. Lowe X, Eskenazi B, Nelson DO, Kidd S, et al. Frequency of XY sperm increases with age in fathers of boys with Klinefelter syndrome. Am J Hum Genet 2001;69:10461054 .

33. Olshan AF, Schnitzer PG, Baird PA. Paternal age and the risk of congenital heart defects. Teratol 1994;50:80-84.

34. Murray L, McCarron P, Bailie K, Middleton R, et al. Association of early life factors and acute lymphoblastic leukaemia in childhood: historical cohort study. Br J Cancer 2002;86:356-361.

35. Cardwell CR, Carson DJ, Patterson CC. Parental age at delivery, birth order, birth weight and gestational age are associated with the risk of childhood Type 1 diabetes: a UK regional retrospective cohort study. Diabet Med 2005;22:200-206.

36. Vestergaard M, Mork A, Madsen KM, Olsen J. Paternal age and epilepsy in the offspring. Eur J Epidemiol 2005;20:1003-1005.

37. Rasmussen F. Paternal age, size at birth, and size in young adulthood—risk factor for schizophrenia. Eur J Endocrin 2006;155:S65-S69.

38. Zammit S, Allebeck P, Dalman C, Lundberg I, et al. Paternal age and risk for schizophrenia. Br J Psychiatr 2003;183:405-408.

39. Tsuchiya KJ, Takagai S, Kawai M, Matsomoto H, et al. Advanced paternal age associated with an elevated risk for schizophrenia in offspring in a Japanese population. Schizophr Res 2005;76:337-342.

40. Reichenberg A, Gross R, Weiser M, Bresnahan M, et al. Advancing paternal age and autism. Arch Gen Psychiatry 2006;63:1026-1032.

41. Croen LA, Najjar DV, Fireman B, Grether JK. Maternal and paternal age and risk of autism spectrum disorders. Arch Pediatr Adolesc Med 2007;161:334-340.

42. Choi JY, Lee KM, Park SK, Noh D-Y, et al. Association of paternal age at birth and the risk of breast cancer in offspring: a case control study. BMC Cancer 2005;5:143.

43. Zhang Y, Kreger BE, Dorgan JF, Cupples LA, et al. Parental age at child's birth and son's risk of prostate cancer. The Framingham study. Am J Epidemiol 1999;150: $1208-1212$.

44. Montgomery SM, Lambe M, Olsson T, Ekbom A. Parental age, family size, and risk of multiple sclerosis. Epidemiology 2004;15:717-723.

45. Slama R, Bouyer J, Windham G, Fenster L, et al. Influence of paternal age on the risk of spontaneous abortion. Am J Epidemiol 2005;161:816-823

46. Kleinhaus K, Perrin M, Friedlander Y, Paltiel O, et al. Paternal age and spontaneous abortion. Obstet Gynecol 2006;108:369-377.

47. De La Rochebrochard E, Thonneau P. Paternal age $>$ or $=40$ years: an important risk factor for infertility. Am J Obstet Gynecol 2003;189:901-905.

48. Reichman NE, Teitler JO. Paternal age as a risk factor for low birthweight. Am J Public Health 2006;96:862-866.

49. Harlap S, Paltiel O, Deutsch L, Knaanie A, et al. Paternal age and preeclampsia. Epidemiology 2002;13:660-667. 